\title{
Quantum gravity corrections for Schwarzschild black holes
}

\author{
Katrin Becker ${ }^{*}$ and Melanie Becker ${ }^{\dagger}$ \\ California Institute of Technology 452-48, Pasadena, California 91125 \\ (Received 17 November 1998; published 14 June 1999)
}

\begin{abstract}
We consider the matrix theory proposal describing 11-dimensional Schwarzschild black holes. We argue that the Newtonian potential between two black holes receives a genuine long-range quantum gravity correction, which is finite and can be computed from the supergravity point of view. In this large-distance limit, the black hole will be treated as a massive pointlike object. The supergravity result agrees with matrix theory up to a numerical factor which we have not computed. [S0556-2821(99)07812-1]
\end{abstract}

PACS number(s): 11.25.Sq, 04.65.+e

\section{INTRODUCTION}

Matrix theory [1] is the nonperturbative formulation of the 11-dimensional $M$ theory. The nonperturbative nature of black hole physics indicates that matrix theory might be an ideal framework to address questions in black hole physics. A concrete model describing Schwarzschild black holes in diverse dimensions was proposed by Horowitz and Martinec [2] and Banks et al. [3] which describes the black hole in terms of a Bolzmann gas of distinguishable D0-branes. This model reproduces correctly the Bekenstein-Hawking entropy, ${ }^{1}$ the size and mass, as well as the leading term of the static Newtonian potential between two black holes. Liu and Tseytlin [5] have generalized this picture to include all-loop large- $N$ supersymmetric Yang-Mills (SYM) corrections or, equivalently, corrections from general relativity.

In this paper we shall be interested in the Newton potential between a pair of Schwarzschild black holes described by the model $[2,3,5]$. Our main interest is the existence of a genuine quantum gravity correction to the gravitational potential, which is finite and can be computed from the supergravity point of view. It is the leading long-range quantum gravity correction that can be computed from matrix theory as well. Happily, we will see that both results indeed agree up to a numerical factor that we have not computed.

The existence of finite quantum gravity corrections to the four-dimensional Newtonian potential has been emphasized by Donoghue [6]. In a series of interesting papers, he showed that the leading long-distance quantum gravity correction to Newton's potential can be reliably calculated in a quantum theory of gravity. Let us briefly review the general idea.

The leading term of the four-dimensional Newtonian potential for two particles with mass $M_{1}$ and $M_{2}$ separated by a distance $r$ is given by

$$
V(r)=-G \frac{M_{1} M_{2}}{r},
$$

\footnotetext{
*Email address: beckerk@ theory.caltech.edu

${ }^{\dagger}$ Email address: mbecker@theory.caltech.edu

${ }^{1} \mathrm{~A}$ different proposal for a microscopic derivation of the Bekenstein-Hawking entropy for Schwarzschild black holes is presented in [4].
}

where $G$ is Newton's constant. This expression is of course only approximately valid. For large masses or large velocities, there are relativistic corrections which can be computed in a post-Newtonian expansion and have been verified experimentally. For a small test particle $M_{2}$, this can be seen from the expansion of the time component of the Schwarzschild metric:

$$
g_{00}=\frac{1-G M_{1} / r c^{2}}{1+G M_{1} / r c^{2}} \approx 1-\frac{2 G M_{1}}{r c^{2}}\left(1-\frac{G M_{1}}{r c^{2}}+\cdots\right) .
$$

In a quantum theory of gravity, we expect that the potential will be corrected by quantum effects. It is well known that when trying to unify quantum mechanics and general relativity we will face the problem that this is a nonrenormalizable theory. Although one can quantize the theory on smooth enough backgrounds, the divergences appearing in particular diagrams are such that they cannot be absorbed into the coupling constants of a minimal general relativity. If one introduces new coupling constants to absorb the divergences, one is led to an infinite number of free parameters and thus to a lack of predictivity. Despite this situation, the leading long-distance quantum corrections can be reliably calculated in "quantum" general relativity.

To leading order in the distance, two massive objects will interact through a Newtonian potential of the form

$$
V(r)=-\frac{G M_{1} M_{2}}{r}\left(1+a \frac{G\left(M_{1}+M_{2}\right)}{r c^{2}}+b \frac{G \hbar}{r^{2} c^{3}}+\cdots\right),
$$

where $a$ and $b$ are some finite numerical coefficients. The first correction is due to general relativity which is the term appearing in Eq. (1.2). The correction proportional to $\hbar$ is a true quantum gravity effect. Its overall form can be fixed by dimensional analysis, while the numerical coefficient can be calculated if we treat gravity as an effective field theory where we can make an expansion in the energy. This procedure is familiar from chiral perturbation theory, which represents the low-energy limit of QCD.

The action describing gravity is then organized in terms of powers of the curvature 


$$
S_{\mathrm{grav}}=\int d^{4} x \sqrt{-g}\left(\frac{2}{\kappa^{2}} R+\alpha R^{2}+\cdots\right),
$$

where $\kappa^{2} \sim G$ and $\alpha$ is a constant. Derivatives are related to the momentum $\partial_{\mu} \sim p_{\mu}$, so that the curvature is of order $p^{2}$, two powers of curvature are of order $p^{4}$, and so on. At low energies or equivalently, long distances terms of order $p^{4}$ can be ignored compared terms of order $p^{2}$.

The quantum gravity correction (1.3) is the leading longdistance quantum gravity effect which is due to the longrange propagation of massless particles such as the graviton. Only the lowest-order coupling contained in the Einstein action is needed to describe this effect since higher-order interactions such as $R^{2}+\cdots$ contain too many powers of the momentum and are neglible at large distances. Since this quantum correction to Eq. (1.3) is finite, it might be the first and simplest quantum gravity corrections that could actually be tested by matrix theory. This is the purpose of our paper.

There are certainly many contributions to the effective action in a quantum theory of gravity that are divergent such as, for example, the $R^{4}$ contribution considered in [7]. This is not the effect that we would like to consider here. Matrix theory regularizes the divergent contributions in $\mathrm{M}$ theory by fixing the value of the cutoff, and there is no finite result on the gravity side with which one could compare.

Let us now come to the matrix theory version of the story. A number of important papers suggested that Schwarzschild black holes could be described in string theory and matrix theory [8]. Horowitz and Martinec [2] and Banks et al. [3] have proposed a beautiful model whose aim is to describe Schwarzschild black holes in diverse dimensions. Many interesting features of black hole physics are correctly reproduced by this model. The basic idea is that Schwarzschild black holes in diverse dimensions can be described in terms of a system of D0-branes in toroidally compactified space. We shall be interested in the 11-dimensional case. One might have thought that this case is especially difficult, because very little is known about the SYM theory for general $N$. However, as we shall see, enough is known to determine the quantum gravity correction we are interested in.

In this paper we would like to see if the matrix theory description is able to reproduce the leading long-distance quantum gravity correction to Newton's potential of an 11dimensional Schwarzschild black hole. We will use crude methods in the sense that we will not compute any numerical coefficients. All the dependences turn out to work out correctly so that precise agreement between matrix theory and supergravity can be found.

This paper is organized as follows. In Sec. II we consider the matrix model point of view. We recapitulate the result for the leading term of Newton's potential and compute relativistic corrections. We see that the leading long-distance quantum gravity effect follows from a one-loop term in the matrix model. In Sec. III we consider the supergravity side of the story, which we then compare with matrix theory. In Sec. IV we present our conclusions. Finally, we would like to remark that in the present paper we are not probing any nonperturbative aspects of black holes. These become relevant, for example, if one would like to determine the end point of black hole evaporation. This is not the purpose of our paper. We would like to test quantum gravity corrections in matrix theory. The leading quantum gravity correction (which is relevant at weak coupling) is the ideal test object as it can be computed in supergravity as well. In this large-distance limit we will treat the black hole as a massive pointlike object which an observer sees when he is very far away.

\section{NEWTONIAN POTENTIAL FROM MATRIX THEORY}

Let us start by reviewing some relevant features of the matrix theory model for Schwarzschild black holes. From now on we will only take into account general dependences, but not any numerical coefficients. The basic idea of [2] and [3] is that a $D$-dimensional Schwarzschild black hole can be described in terms of a Boltzmann gas of distinguishable D0-branes with two-body interactions given by the leading term in the one-loop SYM effective action compactified on $T^{d}(D=11-d)$. We will be interested in the case $D=11$ where the Lagrangian is

$$
L_{\mathrm{eff}}=\frac{N v^{2}}{R}+\frac{G_{11} N^{2}}{R^{3}} \frac{v^{4}}{r^{7}},
$$

where $G_{11}$ is the 11-dimensional Newton's constant, $R$ is the radius of the 11th dimension, $N$ is the number of D0-branes, and $v$ and $r$ are the relative velocity and distance, respectively. This Lagrangian has a holographic scaling property [1]. We would like to rescale the relative transverse coordinate $r$ and the time coordinate $t$ as

$$
r \rightarrow N^{1 / 9} r, \quad t \rightarrow \frac{1}{R} N^{2 / 9} t .
$$

From Eqs. (2.2) it follows that the velocity gets rescaled as

$$
v \rightarrow R\left(N G_{11}\right)^{-1 / 9} v .
$$

The dependence on Newton's constant in formula (2.3) can be determined by dimensional analysis. The Lagrangian (2.1) becomes (up to an overall factor) independent of $N$ and $R$. Applying a qualitative analysis based on the virial theorem, i.e., equating the kinetic and potential term of Eq. (2.1), one can derive a relation between $N$ and the size of the bound state:

$$
G_{11}^{-1} R_{s}^{9}=N .
$$

Since one is treating the D0-brane as a Boltzmann gas, its entropy is of order $N$ so that the above relation is precisely the Bekenstein-Hawking area law for a Schwarzschild black hole [2,3,9], where $R_{s}$ corresponds to the Schwarzschild radius. Using the standard relation between the light cone energy and mass, one can determine the scaling of the mass $M$ as a function of $N$

$$
M \sim G_{11}^{-1 / 9} N^{8 / 9} .
$$

This means that the matrix model correctly reproduces the scaling of the mass in terms of the Schwarzschild radius. 
Finally, one can show that the static Newtonian potential between two equal-mass black holes can be be completely understood in terms of the velocity-dependent potential between the D0-branes. For this purpose we use the connection between the light cone energy and the rest mass plus the potential (2.1) and scaling relations that we just mentioned. The result for the (leading term of the) 11-dimensional Newton potential is [3]

$$
V(r) \sim G_{11} \frac{M^{2}}{r^{7}}\left(G_{11} N\right)^{-1 / 9}=G_{10} \frac{M^{2}}{r^{7}} .
$$

The last factor on the left-hand side of Eq. (2.6) comes from averaging over the longitudinal direction. This is because this factor is $1 / R_{S}$, and if one is working for $S \sim N$, then one can set $R_{S} \sim R$. The reason for this is that the longitudinal box expands in such a way that the black hole fits into it (see the discussion in [3] and [2]). We have further used $G_{10}$ $\sim G_{11} / R$. Notice that the quantity that is being computed here is the 11-dimensional Newtonian potential averaged over the 11 to 10 circle. This is the reason why the result depends on $G_{11} / R$. The averaging over the longitudinal direction introduces the additional factor $1 / R$. Furthermore, notice that in order to compute a long-distance effect such as the leading term of Newton's potential, one can effectively treat the black holes as two massive pointlike objects. As we will be interested only in leading long-distance effects, we shall treat the black holes as two very massive pointlike objects from now on. In the following we would like to compute the leading long-distance corrections of the Newtonian potential, which is averaged over the longitudinal circle.

Let us now discuss general relativity corrections to the previous gravitational potential. Liu and Tseytlin [5] have proposed a generalization of the action (2.1) which includes all loop large- $N$ SYM corrections. The basic idea is to consider the classical Born-Infeld action for a D0-brane probe moving in a supergravity background produced by a D0brane source. The all-loop large- $N$ SYM corrections to Eq. (2.1) can be obtained if one formally extrapolates this action to the short-distance or near-horizon region. The Lagrangian obtained in this way is

$$
L_{\text {eff }}=\frac{r^{7} R}{G_{11}}\left[\sqrt{1-G_{11} \frac{N}{R^{2}} \frac{v^{2}}{r^{7}}-1}\right] .
$$

Expanding Eq. (2.7), one gets Eq. (2.1) plus corrections

$$
L_{\mathrm{eff}}=\frac{N v^{2}}{R}+\frac{G_{11} N^{2}}{R^{3}} \frac{v^{4}}{r^{7}}+\frac{G_{11}^{2} N^{3}}{R^{5}} \frac{v^{6}}{r^{14}}+\cdots .
$$

Let us consider the third term appearing in Eq. (2.8). If we follow the same steps as for the leading contribution, we conclude that this expression corrects the gravitational potential in the following way:

$$
\Delta V(r) \sim G_{10}^{2} \frac{M^{3}}{r^{14}}
$$

This is precisely the form of a relativistic correction to Newton's potential in $D=11$ as can be seen, for example, by analogy to the four-dimensional formula (1.3) that we mentioned in the Introduction. ${ }^{2}$ By the same argument it is easy to see that higher-order corrections in Eq. (2.8) reproduce the correct form of higher-order relativistic corrections to Newton's potential.

Let us now come to our main point: the leading longdistance quantum gravity correction. By now it is well known that the effective action of matrix theory at a given number of loops is a double expansion in the relative velocity and distance between D0-branes. The leading one-loop term, i.e., the $v^{4}$ term appearing in Eq. (2.1), is not renormalized at higher loops $[10,11]$. The $v^{6}$ term has a very special property: it vanishes at one loop, and it is not renormalized beyond two loops [12]. From these considerations we come to the conclusion that the leading long-distance quantum gravity correction to the one-loop $v^{4}$ term corresponds to the $v^{8}$ term at one loop. Let us have a closer look at this contribution.

From the systematic expansion derived in [13], the concrete form of this term is

$$
\Delta L_{\mathrm{eff}}=\frac{N^{2}}{R^{7} M_{\mathrm{Pl}}^{21}} \frac{v^{8}}{r^{15}}=G_{10}^{2} \frac{N^{2}}{M_{\mathrm{Pl}}^{3} R^{5}} \frac{v^{8}}{r^{15}},
$$

where $M_{\mathrm{Pl}}$ is the 11-dimensional Planck mass. We would like to add this correction to the Lagrangian (2.1) and compute the result for the gravitational interaction. Using the same formulas that we used for the leading term and the relativistic corrections, we obtain

$$
V(r) \sim G_{10}^{2} \frac{1}{R^{3} M_{\mathrm{Pl}}^{3}} \frac{M^{2}}{r^{15}} .
$$

We can rewrite this expression in terms of the string coupling constant $g_{s}$ and the ten-dimensional gravitational constant. Recall that the 11-dimensional Planck length is related to the string coupling constant and $\alpha^{\prime}$ as

$$
l_{\mathrm{Pl}}=\left(2 \pi g_{s}\right)^{1 / 3} \sqrt{\alpha^{\prime}},
$$

while the compactification radius is

$$
R=g_{s} \sqrt{\alpha^{\prime}}
$$

This means that the string coupling constant can be expressed in terms of $R$ and $M_{\mathrm{Pl}}$ as

$$
g_{s}^{2}=\frac{2 \pi R^{3}}{l_{\mathrm{Pl}}^{3}}=2 \pi R^{3} M_{\mathrm{Pl}}^{3} .
$$

From this it follows that

$$
G_{10} \sim \frac{G_{11}}{R} \sim g_{s}^{2} \alpha^{\prime 4} .
$$

\footnotetext{
${ }^{2}$ Of course, $c=1$ in string theory conventions.
} 
The dimensionless coupling constant appearing in the correction to Newton's potential (2.11) is nothing but the string coupling constant

$$
V(r) \sim g_{s}^{2} \alpha^{\prime}{ }^{8} \frac{M^{2}}{r^{15}} \sim \frac{G_{10}^{2}}{g_{s}^{2}} \frac{M^{2}}{r^{15}} .
$$

This result looks similar to the finite quantum gravity correction appearing in the four-dimensional formula (1.3). In string theory conventions we can set $\hbar=1$ or equivalently $\hbar$ can be shifted away with a scale tranformation of the metric. This is the origin of the dependence on the string coupling constant in the above formula. We will see this in more detail in the next section. The fact that integer powers of Newton's constant and $g_{s}$ appear indicates that this is a finite correction from the supergravity point of view. Otherwise, fractional powers of $G_{10}$ would be present as a result of the existence of a dimensionful parameter, the cutoff. The above quantum gravity correction is the leading quantum effect as other quantum effects are higher order in $1 / r$.

To summarize, the leading terms of the 11-dimensional Newton's potential from the matrix theory point of view take the form

$$
V(r) \sim \frac{G_{10} M^{2}}{r^{7}}\left(1+\frac{G_{10} M}{r^{7}}+\frac{G_{10}}{g_{s}^{2}} \frac{1}{r^{8}}+\cdots\right) .
$$

This formula has an interesting analogy with Eq. (1.3). The different powers in $r$ are due to the fact that the dimensions of Newton's constant are different in ten and four dimensions. We would like to mention that the matrix model for Schwarzschild black holes will determine many more quantum gravity corrections whose interpretation will have to be understood. In this paper we are only interested in the above quantum gravity correction as this correction can be calculated from the supergravity theory. Let us now have a closer look at the supergravity side of the story.

\section{NEWTONIAN POTENTIAL FROM SUPERGRAVITY}

On the supergravity side we can follow closely the fourdimensional calculation [6] though we will not determine any numerical coefficients. Our aim is to illustrate which Feynman diagrams contribute to the different terms in the gravitational potential.

In ten dimensions two massive objects with mass $M$ separated by a distance $r$ interact to lowest order by the Newtonian potential

$$
V(r) \sim \frac{G_{10} M^{2}}{r^{7}} .
$$

If the mass or velocities of these objects get too big, the potential receives relativistic corrections. These would be of the form

$$
V(r) \sim \frac{G_{10} M^{2}}{r^{7}}\left(1+a \frac{G_{10} M}{r^{7}}+\cdots\right) .
$$

The numerical coefficient $a$ is calculable in a postNewtonian expansion. At some point this expression will be corrected by quantum gravity effects. It is possible to figure out the general form of these corrections using dimensional analysis. Since they arise from loop diagrams, they will involve an additional power of Newton's constant $G_{10}$, and if they are quantum corrections, they will be at least linear in $\hbar$. As we have already mentioned, we will be interested in a very particular quantum gravity correction: the leading long-distance quantum gravity effect. It will be a nonanalytic effect in the momentum transfer, since analytic effects in $q^{2}$ correspond to contact terms in coordinate space. Since this effect is due to long-range propagation of massless particles, the other dimensionful parameter is the distance $r$. The tendimensional Newton's constant has dimension $M^{-1} L^{7}$, and $\hbar$ has dimensions $M L$. The combination

$$
\frac{G_{10} \hbar}{r^{8}}
$$

is dimensionless and provides an expansion parameter for the long-distance quantum effects. Altogether, to leading order we expect a modification of the classical potential of the form

$$
V(r) \sim \frac{G_{10} M^{2}}{r^{7}}\left(1+a \frac{G_{10} M}{r^{7}}+b \frac{G_{10} \hbar}{r^{8}}+\cdots\right) .
$$

The free parameters $a$ and $b$ are finite constants that can be calculated in a quantum theory of gravity by computing Feynman diagrams. Let us illustrate the general idea.

Our starting point is the ten-dimensional Einstein action ${ }^{3}$

$$
S_{\mathrm{grav}} \sim \frac{1}{G_{10}} \int d^{10} x \sqrt{-g} R,
$$

coupled to a massive scalar field

$$
S_{\text {matter }} \sim \int d^{10} x \sqrt{-g}\left(\frac{1}{2} g^{\mu \nu} \partial_{\mu} \varphi \partial_{\nu} \varphi-\frac{1}{2} M^{2} \varphi^{2}\right) .
$$

The quantum fluctuations of the gravitational field $h_{\mu \nu}$ can be expanded around a flat background [14]

$$
g_{\mu \nu}=\eta_{\mu \nu}+\kappa_{10} h_{\mu \nu},
$$

where $\kappa_{10}^{2}=G_{10}$ up to a numerical coefficient. To quantize the fluctuations one needs to fix a gauge and a gauge fixing term has to be added to the Lagrangian. One can then follow the standard rules to quantize the theory by computing Feynman diagrams. We are interested in considering quantum corrections at one loop. For that purpose the Lagrangians have to be expanded to quartic order in the fields.

\footnotetext{
${ }^{3}$ We will not be taking fermions or any other fields of type-IIA supergravity besides the graviton into account. These fields will have to be taken into account to determine the precise numerical coefficients of Eq. (3.4).
} 
The leading term of Newton's potential follows from the single-graviton-exchange diagram where two scalar particles interact through the exchange of a single graviton:

$$
M_{12} \sim \kappa_{10}^{2} T_{\mu \nu}^{(1)}(q) D^{\mu \nu \alpha \beta} T_{\alpha \beta}^{(2)}(-q) .
$$

Here $T_{\mu \nu}$ is the on-shell matrix element of the matter energymomentum tensor and $D^{\mu \nu \alpha \beta}$ is the graviton propagator [15]. The Newton potential can then be found by Fourier transforming:

$$
\frac{1}{M^{2}} M_{12} \sim \frac{G_{10} M^{2}}{q^{2}},
$$

where the factor $1 / M^{2}$ on the left-hand side accounts for the proper normalization. In coordinate space we obtain

$$
V(r) \sim G_{10} \int \frac{d^{9} q}{(2 \pi)^{9}} e^{-i q r} \frac{M^{2}}{q^{2}} \sim \frac{G_{10} M^{2}}{r^{7}} .
$$

To compute one-loop corrections to Newton's potential, we have to consider both vertex corrections and vacuum polarization effects at one loop [6]. Double-graviton-exchange diagrams contribute to this order as well [16]. The form of the contributions originating at one loop can be determined by dimensional analysis, as we shall do in the following. The explicit expression for all the one-loop diagrams contributing to the effect we are interested in can be found in the paper by Hamber and Liu [16]. It will be an important test for matrix theory to actually evaluate the numerical coefficients of the different Feynman diagrams. We reserve this interesting question for a future publication.

The one-loop diagrams will have an additional power of $\kappa_{10}^{2}$ compared to the tree diagram, and $\kappa_{10}^{2}$ has dimension $M^{-8}$. The combination $\kappa_{10}^{2} q^{8}$ is dimensionless. However, loop diagrams will also produce nonanalytic terms of the form $\log \left(-q^{2}\right)$ and $\sqrt{M^{2} /-q^{2}}$, which are dimensionless. ${ }^{4}$ Such nonanalytic effects lead to a power law behavior in coordinate space:

$$
\begin{gathered}
\int \frac{d^{9} q}{(2 \pi)^{9}} e^{-i q r}\left(\sqrt{-q^{2}}\right)^{5} \sim \frac{1}{r^{14}}, \\
\int \frac{d^{9} q}{(2 \pi)^{9}} e^{-i q r}\left(\sqrt{-q^{2}}\right)^{6} \log \left(-q^{2}\right) \sim \frac{1}{r^{15}} .
\end{gathered}
$$

On the other hand, analytic terms in $q^{2}$ correspond to delta functions in coordinate space:

$$
\int \frac{d^{9} q}{(2 \pi)^{9}} e^{-i q r}=\delta^{9}(r) .
$$

We are interested in precisely the terms (3.11) appearing as one-loop effects. The first term will correspond to the

\footnotetext{
${ }^{4}$ The constant which makes the argument of the logarithm dimensionless gives a contact term in coordinate space, which we ignore in our discussion.
}

relativistic correction, while the second term corresponds to the leading quantum correction. In momentum space the contributions to the potential take the form

$$
V(q) \sim G_{10} M^{2}\left[\frac{1}{q^{2}}+G_{10}\left[\left(\sqrt{-q^{2}}\right)^{6} \log \left(-q^{2}\right)+\sqrt{-q^{2}}{ }^{5} M\right]\right],
$$

with some finite numerical coefficients that can be calculated. After Fourier transforming we obtain the Newtonian potential

$$
V(r) \sim \frac{G_{10} M^{2}}{r^{7}}\left(1+a \frac{G_{10} M}{r^{7}}+b \frac{G_{10} \hbar}{r^{8}}+\cdots\right) .
$$

Here we have restored the $\hbar$ dependence by dimensional analysis. This result takes the same form as Eq. (2.17). Of course, in Sec. II we have been using conventions where $\hbar=1$ as one usually does in string theory. We can absorb the value of $\hbar$ in Eq. (3.14) with a rescaling of the metric [17]. This will introduce the dilaton dependence. Consider the action (3.5). Under a scale transformation where lengths are rescaled by a factor $t^{-1}$, the metric transforms as

$$
g_{\mu \nu} \rightarrow t^{-2} g_{\mu \nu} .
$$

Under this transformation the curvature $R_{\mu \nu}$ will remain invariant so that the ten-dimensional action scales as

$$
S \rightarrow t^{-8} S .
$$

At the classical level the normalization of the action is not relevant so that general relativity is scale invariant at this level. When we pass to the quantum theory, we compute a path integral

$$
Z=\int e^{(i / \hbar) S}
$$

The parameter $\hbar$ appearing in this expression can be absorbed into a transformation of the type (3.15). In other words, under a scale transformation $\hbar$ will transform as $\hbar \rightarrow t^{-8} \hbar$. Furthermore, type-IIA supergravity has the same classical scale invariance if the dilaton behaves in the following way under scale transformations:

$$
\phi \rightarrow t^{2} \phi .
$$

From these considerations it follows that the effect of order $\hbar$ that we have been considering corresponds to a $\phi^{-4}$-effect in type-IIA supergravity or, equivalently, a $1 / g_{s}^{2}$ effect. This is what we wanted to show.

One last comment is in order. The fact that one is able to do this calculation from the supergravity point of view using effective field theory techniques is because we are only interested in an effect which is present at very long distances and the black hole is treated as a pointlike massive object. Of course, if one gets to short distances or close to black hole singularities, one would not expect effective field theory to be a valid description. 


\section{CONCLUSIONS}

In this paper we have shown that the matrix model for Schwarzschild black holes of [2] and [3] describes correctly the leading long-range quantum gravity correction to Newton's potential in 11 dimensions. This effect is finite and can be computed from the supergravity point of view from oneloop Feynman diagrams. We have found agreement up to numerical factors that we have not calculated. We have further shown that relativistic corrections to Newton's potential are correctly reproduced. Obviously, it would be of interest to know if it is possible to match the precise numerical coefficients between the Matrix model and supergravity. Since the model of [2] and [3] purports to describe Schwarzschild black holes in diverse dimensions as toroidal compactifications of the 11-dimensional model, it would be interesting to know if the lower-dimensional models also correctly reproduce the finite quantum gravity correction to Newton's potential.

\section{ACKNOWLEDGMENTS}

We would like to thank J. Schwarz for discussions and I. Klebanov for correspondence. This work was supported by the U.S. Department of Energy under Grant No. DE-FG0392-ER40701.
[1] T. Banks, W. Fischler, S. H. Shenker, and L. Susskind, Phys. Rev. D 55, 5112 (1997).

[2] G. T. Horowitz and E. J. Martinec, Phys. Rev. D 57, 4935 (1998).

[3] T. Banks, W. Fischler, I. R. Klebanov, and L. Susskind, J. High Energy Phys. 01, 008 (1998).

[4] K. Sfetsos and K. Skenderis, Nucl. Phys. B517, 179 (1998).

[5] H. Liu and A. Tseytlin, J. High. Energy Phys. 01, 010 (1998).

[6] J. F. Donoghue, Phys. Rev. D 50, 3874 (1994); Phys. Rev. Lett. 72, 2996 (1994).

[7] M. B. Green, M. Gutperle, and P. Vanhove, Phys. Lett. B 409, 177 (1997).

[8] L. Susskind, "Some Speculations about Black Hole Entropy in String theory," hep-th/9309145; G. T. Horowitz and J. Polchinski, Phys. Rev. D 55, 6189 (1997); T. Banks, W. Fischler, I. R. Klebanov, and L. Susskind, Phys. Rev. Lett. 80,
226 (1998); I. R. Klebanov and L. Susskind, Phys. Lett. B 416, 62 (1998).

[9] M. Li, J. High Energy Phys. 01, 009 (1998).

[10] K. Becker and M. Becker, Nucl. Phys. B506, 48 (1997).

[11] S. Paban, S. Sethi, and M. Stern, Nucl. Phys. B534, 137 (1998).

[12] S. Paban, S. Sethi, and M. Stern, J. High Energy Phys. 06, 012 (1998).

[13] K. Becker, M. Becker, J. Polchinski, and A. Tseytlin, Phys. Rev. D 56, 3174 (1997).

[14] G. 't Hooft and M. Veltman, Ann. Inst. Henri Poincaré, Sect. A 20, 69 (1974).

[15] B. S. De Witt, Phys. Rev. 162, 162 (1966).

[16] H. W. Hamber and S. Liu, Phys. Lett. B 357, 51 (1995).

[17] M. B. Green, J. H. Schwarz, and E. Witten, Superstring Theory (Cambridge University Press, Cambridge, England, 1987), Vol. 2, p. 326. 